\title{
TURÁN INEQUALITIES AND ZEROS OF DIRICHLET SERIES ASSOCIATED WITH CERTAIN CUSP FORMS
}

\author{
J. B. CONREY AND A. GHOSH
}

\begin{abstract}
The "Turan inequalities" are a countably infinite set of conditions about the power series coefficients of certain entire functions which are necessary in order for the function to have only real zeros. We give a one-parameter family of generalized Dirichlet series, each with functional equation, for which the Turan inequalities hold for the associated $\xi$-function (normalized so that the critical line is the real axis). For a discrete set of values of the parameter the Dirichlet series has an Euler product and is the $L$-series associated to a modular form. For these we expect the analogue of the Riemann Hypothesis to hold. For the rest of the values of the parameter we do not expect an analogue of the Riemann Hypothesis. We show for one particular value of the parameter that the Dirichlet series in fact has zeros within the region of absolute convergence.
\end{abstract}

\section{RIEMANN'S INTEGRAL REPRESENTATION FOR THE $\xi$-FUNCTION}

One approach to the study of the zeros of the Riemann zeta-function begins with the integral representation for Riemann's $\xi$-function. The $\xi$-function is given by

$$
\xi(s)=\frac{1}{2} s(s-1) \pi^{-s / 2} \Gamma(s / 2) \zeta(s)
$$

where $\zeta$ is Riemann's zeta-function and $s=\sigma+i t$ is a complex variable. The Mellin transform of $\xi$ involves the theta-function:

$$
\theta(z)=\sum_{n=-\infty}^{\infty} e\left(\frac{n^{2} z}{2}\right)
$$

where $e(z)=\exp (2 \pi i z)$. We have

$$
\pi^{-s / 2} \Gamma\left(\frac{s}{2}\right) \zeta(s)=\int_{0}^{\infty} \psi(x) x^{s / 2} \frac{d x}{x}
$$

where

$$
\psi(u)=\sum_{n=1}^{\infty} e^{-\pi n^{2} u}=\frac{\theta(i u)-1}{2} .
$$

Using the transformation formula for $\theta$

$$
\theta(-1 / z)=z^{1 / 2} \theta(z)
$$

Received by the editors April 10, 1992.

1991 Mathematics Subject Classification. Primary 11F66; Secondary 30C15.

Research of the second author supported in part by the Alfred P. Sloan Foundation. Research of both authors supported in part by a grant from the NSF. 
and integration by parts we arrive at Riemann's formula:

$$
\xi\left(\frac{1}{2}+i t\right)=\int_{-\infty}^{\infty} F(u) e^{i u t} d u
$$

where

$$
F(u)=\frac{d}{d u}\left(u^{1 / 4} \psi^{\prime}(u)\right) .
$$

Another expression for $F$ is given by

$$
F(u)=\sum_{n=1}^{\infty}\left(4 \pi^{2} n^{4} e^{9 u / 2}-6 n^{2} \pi e^{5 u / 2}\right) \exp \left(-n^{2} \pi e^{2 u}\right) .
$$

All of the above can be found in Titchmarsh [14].

The idea is to study $F$ and then try to deduce information about the zeros of $\zeta$. There are various conditions known which ensure that an entire function given as a trigonometrical integral has all real zeros. Pólya [11] and de Bruijn [5] have proven some of the most interesting theorems in this area.

\section{Properties of $F$}

What is known about $F$ ? The functional equation

$$
\xi(s)=\xi(1-s)
$$

for the $\xi$-function is equivalent to the fact that $F$ is even. Also it is known that $F(u)$ is positive and decreasing for $u \geq 0$. This was first shown by Wintner [15] and then again by Spira [13]. In 1982 Matiyasevich [10] investigated $F$ and proved the Turán inequalities for the $\xi$-function. These are inequalities involving the power series coefficients of certain real entire functions which must hold if the function has all real zeros. In our case they may be expressed as

$$
b_{m}^{2}>\frac{2 m-1}{2 m+1} b_{m-1} b_{m+1} \quad(m=1,2, \ldots)
$$

where

$$
b_{m}=\int_{0}^{\infty} F(u) u^{2 m} d u
$$

Note that

$$
\Xi(t):=\xi\left(\frac{1}{2}+i t\right)=\sum_{m=0}^{\infty} \frac{(-1)^{m} b_{m}(2 t)^{2 m}}{(2 m) !} .
$$

The problem of proving the Turán inequalities for the Riemann $\Xi$-function was proposed by Pólya as a test for the Riemann Hypothesis. (If the Turán inequalities failed, then the Riemann Hypothesis would be false.) Grosswald [7] showed that the inequalities hold for $\Xi$ for all sufficiently large $m$. In 1986 Csordas, Norfolk, and Varga [3], not knowing about Matiyasevich's paper, ${ }^{1}$ gave a different proof of the Turán inequalities for all $m$.

The proofs of the above properties of $F$ are somewhat complicated in that they involve a lot of numerical calculations. Much of the difficulty arises from

\footnotetext{
${ }^{1}$ We thank Professor G. Warner for bringing Matiyasevich's paper to our attention.
} 
the complicated form of $F$ given above which is essentially due to the pole of $\zeta(s)$ at $s=1$.

\section{The Ramanujan taU-FunCtion}

The Dirichlet series

$$
L(s)=\sum_{n=1}^{\infty} \frac{\tau(n)}{n^{s}}
$$

where $\tau$ is Ramanujan's tau-function (defined below) has properties similar to those of the Riemann zeta-function and has some additional nice features. One is that $L(s)$ is an entire function. Another is that the Mellin transform of $\xi_{\tau}$, the associated " $\xi$-function", has a simple infinite product expansion. This product expansion is not typical in the study of Dirichlet series which are expected to satisfy the Riemann Hypothesis. Nevertheless it is interesting to see what sort of information can be gained from the use of the infinite product. In particular, the above properties of $F$ can be proven in a fairly straightforward manner for the Fourier transform $F_{\tau}$ of $\xi_{\tau}(6+i t)$, the "6-line" being the critical line for this function.

Ramanujan's tau function may be defined by equating coefficients of the power series on both sides of

$$
\sum_{n=1}^{\infty} \tau(n) x^{n}=x \prod_{n=1}^{\infty}\left(1-x^{n}\right)^{24}
$$

The associated Dirichlet series is

$$
L(s)=L_{\tau}(s)=\sum_{n=1}^{\infty} \tau(n) n^{-s} .
$$

This series is absolutely convergent for $\sigma=\Re s>13 / 2$. The $\xi$-function for $\tau$ is given by

$$
\xi_{\tau}(s)=(2 \pi)^{-s} \Gamma(s) L(s)
$$

and it satisfies the functional equation

$$
\xi_{\tau}(s)=\xi_{\tau}(12-s)
$$

This functional equation is equivalent to the fact that

$$
\Delta(z)=\sum_{n=1}^{\infty} \tau(n) e(n z)
$$

is a holomorphic cusp form of weight 12 for the full modular group which in turn is equivalent to (i) $\Delta(z)$ is expressible in terms of a Fourier series in $z$ in which coefficients of $e(n z)$ with $n \leq 0$ vanish, and (ii) $\Delta$ satisfies the transformation formula

$$
\Delta(-1 / z)=z^{12} \Delta(z) .
$$

(See Hardy [9, Chapter X] for introductory information about $\tau$.)

Now

$$
\xi_{\tau}(s)=\int_{0}^{\infty} \Delta(i y) y^{s} \frac{d y}{y}
$$


so that

$$
\Xi_{\tau}(t)=\xi_{\tau}(6+i t)=\int_{-\infty}^{\infty} \Delta\left(i e^{u}\right) e^{6 u} e^{i u t} d u
$$

is an entire even function of $t$. We define

$$
F_{\tau}(u)=\Delta\left(i e^{u}\right) e^{6 u}
$$

\section{Properties of $F_{\tau}$}

We see that $F_{\tau}(u)$ is an even function of $u$ by the functional equation for $\xi_{\tau}$. The fact that $F_{\tau}(u)>0$ for real $u$ is immediately obvious from the product formula for $\Delta$ :

$$
F_{\tau}(u)=e^{6 u} e^{-2 \pi e^{u}} \prod_{n=1}^{\infty}\left(1-e^{-2 \pi n e^{u}}\right)^{24}
$$

We can also see that $F_{\tau}(u)$ is decreasing for positive $u$ by calculating the logarithmic derivative. We first observe that

$$
y \frac{d}{d y} \sum_{n=1}^{\infty} \log \left(1-y^{n}\right)=-y \frac{d}{d y} \sum_{m, n=1}^{\infty} \frac{y^{m n}}{m}=-\sum_{m, n=1}^{\infty} n y^{m n}=-\sum_{n=1}^{\infty} \sigma(n) y^{n}
$$

where

$$
\sigma(n)=\sum_{d \mid n} d
$$

is the sum of divisors of $n$. Let $x=2 \pi e^{u}$ and $y=e^{-x}$. Then

$$
F_{\tau}(u)=e^{6 u} y \prod_{n=1}^{\infty}\left(1-y^{n}\right)^{24}
$$

so that

$$
\frac{F_{\tau}^{\prime}}{F_{\tau}}(u)=6+\left(\frac{1}{y}-24 \sum_{n=1}^{\infty} \sigma(n) y^{n}\right) \frac{d y}{d u}=6-x\left(1-\Sigma_{0}(x)\right)
$$

where

$$
\Sigma_{k}(x)=24 \sum_{n=1}^{\infty} n^{k} \sigma(n) y^{n}
$$

(The expansion of $F^{\prime} / F$ above is related to the Fourier expansion of the Eisenstein series $E_{2}$ :

$$
E_{2}(z)=1-24 \sum_{n=1}^{\infty} \sigma(n) e(n z)
$$

$E_{2}$ is not a modular form of weight 2 ; it transforms according to the formulae

$$
E_{2}(-1 / z)=z^{2} E_{2}(z)+12 z / 2 \pi i
$$

and $E_{2}(z+1)=E_{2}(z)$.)

Now $F_{\tau}^{\prime} / F_{\tau}$ is an odd function of $u$ so that $F_{\tau}^{\prime} / F_{\tau}(0)=0$. Thus, to show that $F_{\tau}^{\prime}(u)<0$ for $u>0$ it suffices to prove that $\left(F_{\tau}^{\prime} / F_{\tau}\right)^{\prime}(u)<0$ for $u>0$. But

$$
\left(\frac{F_{\tau}^{\prime}}{F_{\tau}}\right)^{\prime}(u)=\left(-1+\Sigma_{0}(x)+x \Sigma_{0}^{\prime}(x)\right) \frac{d x}{d u}=-x+x \Sigma_{0}(x)-x^{2} \Sigma_{1}(x),
$$


since $\Sigma_{k}^{\prime}(x)=-\Sigma_{k+1}(x)$, so that the above is

$$
=-x\left(1-24 \sum_{n=1}^{\infty} \sigma(n) y^{n}(1-n x)\right) .
$$

Since $u \geq 0$ corresponds to $x \geq 2 \pi$, each of the terms $1-n x<0$ so that the whole expression is negative.

Arguing in the same way we see that $\left(F_{\tau}^{\prime} / F_{\tau}\right)^{\prime \prime}(u)$ is odd and

$$
\begin{aligned}
\left(\frac{F_{\tau}^{\prime}}{F_{\tau}}\right)^{\prime \prime \prime}(u) & =-x\left(x^{3} \Sigma_{3}(x)-6 x^{2} \Sigma_{2}(x)+7 x \Sigma_{1}(x)-\Sigma_{0}(x)+1\right) \\
& =-x\left(1-24 \sum_{n=1}^{\infty} \sigma(n) y^{n} P_{3}(n x)\right)
\end{aligned}
$$

where

$$
P_{3}(x)=1-7 x+6 x^{2}-x^{3}<0
$$

for $x>6$. Thus we conclude that $\left(F_{\tau}^{\prime} / F_{\tau}\right)^{\prime \prime}(u)<0$ for $u>0$, i.e., that $F_{\tau}^{\prime} / F_{\tau}$ is concave for $u>0$. $\Xi_{\tau}$

The above considerations will be shown to imply the Turán inequalities for

\section{A FAMILY OF DiRICHLET SERIES}

In this section we introduce a family of (generalized) Dirichlet series based on the example of the last section. We will denote the Dirichlet series by $A_{k}(s)$. The associated entire functions $\xi_{k}(s)=\gamma_{k}(s) A_{k}(s)$ have functional equations

$$
\xi_{k}(s)=\xi_{k}(k / 2-s) \text {. }
$$

Here $k>0$ is a real parameter. These functions have simple expressions

$$
\Xi_{k}(t):=\xi\left(\frac{k}{4}+i t\right)=\int_{-\infty}^{\infty} e^{-k g(u)} e^{i u t} d u
$$

as trigonometric integrals. The main properties of $\xi_{k}$ are

(1) the functions

$$
\Xi_{k}(t)=\xi_{k}(k / 4+i t)
$$

satisfy the Turán inequalities;

(2) for all $k$ there are $\gg T$ zeros on the critical line $\sigma=k / 4$ up to a height $T$;

(3) for $k=1,2,3,4,6,8,12,24$ a positive proportion of zeros of $A_{k}$ are on the line $\sigma=k / 4$;

(4) for all other $k$ there are infinitely many complex zeros outside the critical strip.

The properties (2)-(4) are reminiscent of the properties of the Epstein zetafunctions (or of the Eisenstein series). Property (1) was our original motivation for studying these functions. We remark that Alter [1] has used Grosswald's method to show that the Turán inequalities for large $m$ are satisfied for various $\Xi$-functions that arise in number theory, including $\Xi_{\tau}$. 
While the Turán inequalities are necessary conditions for our $\Xi$-functions to have all real zeros, these examples show that they are not sufficient as they also hold for many $\Xi$-functions which do not satisfy the Riemann Hypothesis.

\section{Definition of $\xi_{k}$}

We now proceed to define our one parameter family of $\xi$-functions. Let

$$
\prod_{n=1}^{\infty}\left(1-x^{n}\right)^{k}=\sum_{n=0}^{\infty} p_{k}(n) x^{n}
$$

and let

$$
A_{k}(s)=\sum_{n=0}^{\infty} \frac{p_{k}(n)}{(n+k / 24)^{s}} .
$$

We note that with $k=1$, by Euler's pentagonal number formula,

$$
A_{1}(s)=24^{-s} L\left(2 s, \chi_{12}\right)
$$

where $\chi_{12}$ is the unique primitive real quadratic Dirichlet character modulo 12. With $k=24$,

$$
A(s)=\sum_{n=1}^{\infty} \tau(n) n^{-s}
$$

where $\tau$ is Ramanujan's tau-function. We observe that

$$
\prod_{n=1}^{\infty}\left(1-e^{-2 \pi n v}\right)=\exp \left(-\sum_{n=1}^{\infty} \sigma_{-1}(n) \exp (-2 \pi n v)\right) .
$$

This product is closely related to the Dedekind eta-function:

$$
\eta(z)=e\left(\frac{z}{24}\right) \prod_{n=1}^{\infty}(1-e(n z))
$$

which satisfies the transformation formula

$$
\eta(-1 / z)=\sqrt{z / i} \eta(z) .
$$

Of course,

$$
\Delta(z)=\eta(z)^{24}
$$

Now let

$$
g(u)=-\frac{u}{4}+\frac{\pi e^{u}}{12}+\sum_{n=1}^{\infty} \sigma_{-1}(n) e^{-2 \pi n e^{u}}
$$

where, as usual, $\sigma_{a}(n)$ is the sum of the $a$ th powers of positive divisors of $n$. Then we define

$$
\Xi_{k}(t)=\int_{-\infty}^{\infty} e^{k g(u)+i u t} d t .
$$

Now $g$ is an even function, by the transformation formula for the Dedekind eta-function so that $\Xi_{k}$ is an even entire function. We have

$$
\Xi_{k}(t)=\int_{0}^{\infty} v^{k / 4+i t} e^{-\pi v k / 12} \sum_{n=0}^{\infty} p_{k}(n) \exp (-2 \pi n v) \frac{d v}{v} .
$$


If we integrate term-by-term and use the substitution $2 \pi n v=w$ we get, with $s=k / 4+i t$,

$$
\xi_{k}(s)=(2 \pi)^{-s} \Gamma(s) \sum_{n=0}^{\infty} \frac{p_{k}(n)}{(n+k / 24)^{s}}
$$

where

$$
\xi_{k}(k / 4+i t)=\Xi_{k}(t)
$$

Now $\Xi_{k}(t)=\Xi_{k}(-t)$ implies that

$$
\xi_{k}(s)=\xi_{k}(k / 2-s) .
$$

\section{The TURÁN INEQUALITIES FOR $\Xi_{k}$}

Regarding the Turán inequalities, we have the following result.

Theorem 1. Let $\phi \in C^{3}(\mathbf{R})$. Let $\phi(u)$ be positive, even, and decreasing for positive $u$, and suppose that $\phi^{\prime} / \phi$ is decreasing and concave for $u>0$. Suppose that $\phi$ is rapidly decreasing so that

$$
X(t)=\int_{-\infty}^{\infty} \phi(u) e^{i t u} d u
$$

is an entire function of $t$. Then $X(t)$ satisfies the Turán inequalities.

Proof. From our hypotheses we can prove the Turán inequalities either by the method of Csordas, Norfolk, and Varga [3] or by the method of Matiyasevich [10]. We will show both of these.

First we follow the proof in [3]. The main area of interest is Proposition 2.4 of that paper where it is shown (see also (2.28)) that

$$
\lambda_{x}=\frac{2}{\Gamma(x+2)} \int_{0}^{\infty} u^{2 x+3} \phi(u) d u
$$

has the property that $\log \lambda_{x}$ is concave on $(-1, \infty)$. In Proposition 2.5 , it is shown that this property of $\lambda_{x}$ implies the Turán inequalities for $m \geq 2$. We will show that $\log \lambda_{x}$ is concave for $x \geq-2$ which will imply the Turán inequalities for $m \geq 1$, thus avoiding the numerical verification in the case $m=1$.

Define $\mu_{x}=\lambda_{x-1}$ and $f(t)=\phi(\sqrt{t})$. Then

$$
\mu_{x}=\frac{1}{\Gamma(x+1)} \int_{0}^{\infty} u^{x} f(u) d u .
$$

Now $\mu$ is defined exactly as $\lambda$ in (2.13) of [3] except that $K$ there is replaced by $f$ here. The only property of $K$ that is used in the proof is that $\log K(u)$ is concave on $(0, \infty)$. Thus, we may conclude, exactly as in that proposition, that $\mu_{x}$ is concave on $(-1, \infty)$ provided we show that $\log f(u)$ is concave on $(0, \infty)$. But then $\lambda_{x}$ is concave on $(-2, \infty)$ which is what we want.

Now

$$
f^{\prime}(u)=\phi^{\prime}(\sqrt{u}) / 2 \sqrt{u}
$$

and

$$
f^{\prime \prime}(u)=\frac{\phi^{\prime \prime}(\sqrt{u})-\phi^{\prime}(\sqrt{u}) / \sqrt{u}}{4 u} .
$$


In order to show that $\log f$ is concave, we have to show that $f f^{\prime \prime}(u)<f^{\prime}(u)^{2}$ for $u>0$. Using the above expressions and replacing $\sqrt{u}$ by $u$ this inequality simplifies to

$$
\phi \phi^{\prime \prime}(u)-\phi^{\prime}(u)^{2}<\phi \phi^{\prime}(u) / u \text {. }
$$

Dividing by $\phi^{2}$, this inequality further simplifies to

$$
u\left(\frac{\phi^{\prime}}{\phi}\right)^{\prime}(u)<\frac{\phi^{\prime}}{\phi}(u)
$$

or

$$
u h^{\prime}(u)<h(u)
$$

where $h=\phi^{\prime} / \phi$. At $u=0$ both sides of the inequality are 0 . Therefore, it suffices to show that if $u>0$, then

$$
H(u)=u-\frac{h}{h^{\prime}}(u)
$$

is increasing. (Note that $h(u)<0$ and $h^{\prime}(u)<0$ for $u>0$ by hypothesis.) We observe that

$$
H^{\prime}(u)=\frac{h h^{\prime \prime}}{\left(h^{\prime}\right)^{2}}(u)>0
$$

since $h$ and $h^{\prime \prime}$ are both $<0$.

Now we consider Matiyasevich's proof [10]. There it is sufficient to show that

$$
\left(u \phi^{\prime}(u)\right)^{\prime} \phi^{\prime}(u)-u \phi(u) \phi^{\prime \prime}(u)>0
$$

for all $u>0$. He does this by a machine calculation in the case of the Riemann $\Xi$-function. We observe that upon dividing by $\phi^{2}$ the above inequality is seen to be equivalent to $u h^{\prime}(u)<h(u)$ for $u>0$ where $h=\phi^{\prime} / \phi$. We proved this inequality above.

As a corollary of this theorem and the calculations from the first section we have that the Turán inequalities are satisfied by the $\Xi_{k}$.

\section{ZEROS OF $\xi_{k}$}

Hardy's method (see Titchmarsh [14, Chapter X]) can be used to show that $\xi_{k}$ has $\gg T$ zeros on $\sigma=k / 4$ up to a height $T$.

In the cases $k=1,2,3,4,6,8,12,24$ the Dirichlet series $(k / 24)^{s} A_{k}(s)$ has an Euler product and is the Dirichlet series associated to a Hecke eigenform for a congruence subgroup of $S L(2, Z)$. Hence by the work of Hafner [8] it has a positive proportion of zeros on the critical line.

For the assertions about zeros outside the critical strip we refer to the work of Davenport and Heilbronn [4] and of Cassels [2] on zeros of Epstein zetafunctions and of Hurwitz zeta-functions outside of the critical strip. We will illustrate the method of [4] in the case that $k=48$.

Theorem 2. The function $\xi_{48}(s)$ has infinitely many zeros in the half plane $\sigma>$ $25 / 2$.

Remark. The critical line for $\xi_{48}(s)$ is $\sigma=12$. The critical strip is $23 / 2<$ $\sigma<25 / 2$. 
Proof. The Dirichlet series associated with $\xi_{48}$ is

$$
F(s)=\sum_{n=1}^{\infty} \frac{f(n)}{n^{s}}
$$

where

$$
\sum_{n=1}^{\infty} f(n) e(n z)=\Delta(z)^{2} .
$$

Since $\Delta(z)$ is a cusp form of weight 12 for the full modular group, it follows that $\Delta(z)^{2}$ is a cusp form of weight 24 for the full modular group. It is well known that the space of cusp forms of weight 24 for the full modular group has dimension 2 as a vector space over $\mathbf{C}$. As a basis for this space we can take cusp forms $\sum_{n \geq 1} a(n) e(n z)$ and $\sum_{n \geq 1} b(n) e(n z)$ where $a(n)$ and $b(n)$ are multiplicative arithmetic functions. Let $A(s)=\sum a(n) n^{-s}$ and $B(s)=$ $\sum b(n) n^{-s}$ be the associated Dirichlet series. Now $F(s)$ is a linear combination of $A(s)$ and $B(s)$. Since $a(1)=b(1)=1$ and $f(1)=0$, it follows that

$$
F(s)=C(A(s)-B(s))
$$

for some constant $C$. To make things easier we scale so that the critical strip is $0<\sigma<1$. Thus, let $\alpha(n)=a(n) / n^{23 / 2}$ and $\beta(n)=b(n) / n^{23 / 2}$. Let $G(s)=\sum(\alpha(n)-\beta(n)) n^{-s}$. It is a consequence of Deligne's Theorem that $|\alpha(n)| \leq d(n)$, and similarly for $b(n)$, where $d(n)$ is the number of positive divisors of $n$.

Now the idea is to construct for every $\delta>0$ an auxiliary Dirichlet series $Z(s)=Z_{\delta}(s)$ with the properties

(1) $Z(s)$ has a zero in $1<\sigma<1+\delta$

(2) for every $\epsilon>0$ and every $s_{0}=\sigma_{0}+i t_{0}$ with $\sigma_{0}>1$ there is an $\eta>0$ and a $T$ such that

$$
|Z(s)-G(s+i T)|<\epsilon
$$

for all $s$ in $\left|s-s_{0}\right|<\eta$.

Assuming we can construct such $Z_{\delta}$ it then follows from Rouché's Theorem that $G(s)$ has infinitely many zeros in $\sigma>1$.

We now define $Z_{\delta}$. Assume that

$$
\sum_{p}|\alpha(p)| p^{-1-\delta} \geq \sum_{p}|\beta(p)| p^{-1-\delta}
$$

Otherwise change the roles of $\alpha$ and $\beta$ below. Define

$$
Z(s)=\sum_{n=1}^{\infty} \frac{(\alpha(n)-\beta(n)) \lambda(n)}{n^{s}}
$$

where $\lambda(n)$ is a completely multiplicative arithmetic function defined for primes $p$ by

$$
\lambda(p)= \begin{cases}\operatorname{sgn} \alpha(p) & \text { if } p \leq c(\delta), \\ \operatorname{sgn} \beta(p) & \text { if } p>c(\delta) \text { and } \operatorname{sgn} \beta(p) \neq \operatorname{sgn} \alpha(p), \\ \epsilon_{p} & \text { if } p>c(\delta) \text { and } \operatorname{sgn} \beta(p)=\operatorname{sgn} \alpha(p),\end{cases}
$$


where $c(\delta)$ will be specified later and $\epsilon_{p}$ is a sequence consisting of +1 and -1 chosen in such a way that

$$
\sum_{\substack{p \\ \operatorname{sgn} \alpha(p)=\operatorname{sgn} \beta(p)}} \frac{(\alpha(p)-\beta(p)) \epsilon_{p}}{p}
$$

converges. Also, we make the convention here that $\operatorname{sgn}(0)=1$.

Since the logarithms of the primes are linearly independent, property (2) above follows from Kronecker's theorem by choosing $T$ in such a way that $\left|p^{i T}-\lambda(p)\right|$ is small for all $p$ up to a certain height depending on $s_{0}$ and $\epsilon$.

To verify property (1) we need the following lemma.

Lemma. With $\alpha$ and $\beta$ as above we have the estimate:

$$
\sum_{\substack{p \\ \operatorname{sgn} \alpha(p) \neq \operatorname{sgn} \beta(p)}} \frac{|\beta(p)|}{p^{1+\delta}} \gg \log \frac{1}{\delta}
$$

as $\delta \rightarrow 0^{+}$.

As an immediate consequence of the lemma we have the estimate:

Corollary. As $\delta \rightarrow 0^{+}$,

$$
\sum_{p} \frac{|\beta(p)|-\beta(p) \lambda^{\prime}(p)}{p^{1+\delta}}>>\log \frac{1}{\delta}
$$

where $\lambda^{\prime}$ is a completely multiplicative function for which $\lambda^{\prime}(p)=\operatorname{sgn} \alpha(p)$.

For the sum in the corollary is twice the sum in the lemma.

Assuming the lemma, we will now show that if $c=c(\delta)$ is large enough, then $Z(1+\delta)>0$. First of all,

$$
\sum_{n>c(\delta)} n^{-1-\delta}|\alpha(n)-\beta(n)||\lambda(n)| \ll \sum_{n>c} n^{-1-\delta} d(n) \ll c^{-\delta} \delta^{-1} \log c .
$$

Similarly, since $\lambda(n)=\lambda^{\prime}(n)$ for all $n \leq c(\delta)$,

$$
\sum_{n \leq c(\delta)} \frac{(\alpha(n)-\beta(n)) \lambda(n)}{n^{1+\delta}}=\sum_{n=1}^{\infty} \frac{(\alpha(n)-\beta(n)) \lambda^{\prime}(n)}{n^{1+\delta}}+O\left(c^{-\delta} \delta^{-1} \log c\right) .
$$

Now

$$
S_{1}(\alpha):=\sum_{n=1}^{\infty} \frac{\alpha(n) \lambda^{\prime}(n)}{n^{1+\delta}}=k_{1}(\delta) \sum_{n=1}^{\infty} \frac{|\alpha(n)|}{n^{1+\delta}}=k_{2}(\delta) \prod_{p}\left(1+\frac{|\alpha(p)|}{p^{1+\delta}}\right)
$$

and

$$
S_{2}:=\sum_{n=1}^{\infty} \frac{\beta(n) \lambda^{\prime}(n)}{n^{1+\delta}}=k_{3}(\delta) \prod_{p}\left(1+\frac{\beta(p) \lambda^{\prime}(p)}{p^{1+\delta}}\right)
$$

where $k_{i}(\delta)$ denotes a function for which $1 \ll k_{i}(\delta) \ll 1$ as $\delta \rightarrow 0^{+}$. Thus,

$$
\begin{aligned}
Z(1 & +\delta)=S_{1}(\alpha)-S_{2}+O\left(c^{-\delta} \delta^{-1} \log c\right) \geq S_{1}(\beta)-S_{2}+O\left(c^{-\delta} \delta^{-1} \log c\right) \\
& =k_{4}(\delta) \prod_{p}\left(1+\frac{|\beta(p)|}{p^{1+\delta}}\right)-k_{5}(\delta) \prod_{p}\left(1+\frac{\beta(p) \lambda^{\prime}(p)}{p^{1+\delta}}\right)+O\left(c^{-\delta} \delta^{-1} \log c\right) \\
& =k_{6}(\delta) \exp \left(\sum_{p} \frac{|\beta(p)|}{p^{1+\delta}}\right)-k_{7}(\delta) \exp \left(\sum_{p} \frac{\beta(p) \lambda^{\prime}(p)}{p^{1+\delta}}\right)+O\left(c^{-\delta} \delta^{-1} \log c\right) .
\end{aligned}
$$


If the second sum is $<0$, then, by the lemma,

$$
Z(1+\delta) \gg \exp \left(r \log \frac{1}{\delta}\right)+O\left(c^{-\delta} \delta^{-1} \log c\right) \gg \delta^{-r}
$$

for some $r>0$ provided that $c$ is sufficiently large. If the second sum is $\geq 0$, then

$$
\begin{aligned}
Z(1+\delta) & \gg \exp \sum_{p}\left(\frac{|\beta(p)|-\beta(p) \lambda^{\prime}(p)}{p^{1+\delta}}\right)+O\left(c^{-\delta} \delta^{-1} \log c\right) \\
& \gg \exp \left(r \log \frac{1}{\delta}\right)+O\left(c^{-\delta} \delta^{-1} \log c\right) \gg \delta^{-r}
\end{aligned}
$$

by the corollary. In any event, we conclude that $Z(1+\delta)>0$ provided that $c(\delta)$ is large enough. On the other hand, it follows from the lemma that if $\sigma \rightarrow 1^{+}$, then $Z(\sigma) \rightarrow-\infty$ since, by definition of $\lambda$,

$$
\begin{aligned}
\sum_{p} \frac{(\alpha(p)-\beta(p)) \lambda(p)}{p^{\sigma}} & =\sum_{\operatorname{sgn} \alpha(p) \neq \operatorname{sgn} \beta(p)} \frac{(\alpha(p)-\beta(p)) \lambda(p)}{p^{\sigma}}+O\left(c^{-\delta} \delta^{-1} \log c\right) \\
& <\sum_{\operatorname{sgn} \alpha(p) \neq \operatorname{sgn} \beta(p)} \frac{|\beta(p)|}{p^{\sigma}}+O\left(c^{-\delta} \delta^{-1} \log c\right) \rightarrow-\infty
\end{aligned}
$$

by part (2) of the lemma. Therefore $Z(s)$ has a zero in $1<\sigma<1+\delta$ as desired.

\section{Proof of LemMa}

It only remains to prove the lemma. We have

$$
\sum_{\substack{p \\(p) \neq \operatorname{sgn} \beta(p)}} \frac{|\beta(p)|}{p^{1+\delta}} \geq \frac{1}{2} \sum_{\substack{p \\ \operatorname{sgn} \alpha(p) \neq \operatorname{sgn} \beta(p)}} \frac{|\alpha(p) \beta(p)|}{p^{1+\delta}} .
$$

Now by the Rankin-Selberg method.

$$
\sum_{p} \frac{\alpha(p) \beta(p)}{p^{1+\delta}}=O(1)
$$

as $\delta \rightarrow 0^{+}$. On the other hand,

$$
\sum_{p} \frac{\alpha(p) \beta(p)}{p^{1+\delta}}=\sum_{\substack{p \\ \operatorname{sgn} \alpha(p)=\operatorname{sgn} \beta(p)}} \frac{|\alpha(p) \beta(p)|}{p^{1+\delta}}-\sum_{\substack{p \\ \operatorname{sgn} \alpha(p) \neq \operatorname{sgn} \beta(p)}} \frac{|\alpha(p) \beta(p)|}{p^{1+\delta}} .
$$

Therefore,

$$
\sum_{\substack{p \\ \operatorname{sgn} \alpha(p) \neq \operatorname{sgn} \beta(p)}} \frac{|\alpha(p) \beta(p)|}{p^{1+\delta}}=\frac{1}{2} \sum_{p} \frac{|\alpha(p) \beta(p)|}{p^{1+\delta}}+O(1) .
$$

Now we use Hölder's inequality to get a lower bound for the latter sum. Thus, if

$$
r_{p}=|\alpha(p) \beta(p)| / p^{1+\delta},
$$


then

$$
\sum_{p} r_{p} \geq \frac{\left(\sum_{p} r_{p}^{2}\right)^{3 / 2}}{\left(\sum_{p} r_{p}^{4}\right)^{1 / 2}}
$$

Now $\sum_{n} \alpha(n)^{2} \beta(n)^{2} / n^{s}$ has a simple pole at $s=1$ by the work of Shahidi [12]. Therefore, it easily follows that

$$
\sum_{p} \frac{r_{p}^{2}}{p^{1+\delta}} \sim \log \frac{1}{\delta}
$$

as $\delta \rightarrow 0^{+}$. We estimate the denominator trivially by

$$
\sum_{p} \frac{r_{p}^{4}}{p^{1+\delta}} \leq 256 \sum_{p} p^{-1-\delta} \ll \log \frac{1}{\delta} .
$$

Thus the lemma follows.

\section{CONCLUSION}

In 1914 Grommer [6] gave an infinite sequence of sets of inequalities that the Taylor coefficients of an entire function $f$ with all real zeros must satisfy. The first set of inequalities in this sequence are equivalent to the Turán inequalities. Thus, the Turán inequalities are really the first Grommer inequalities. Grommer showed that all of his inequalities taken together represent a necessary and sufficient condition for $f$ to have all real zeros. It would be interesting to investigate whether the class of functions we have described in this paper satisfy the second set of Grommer inequalities.

\section{REFERENCES}

1. R. Alter, On a necessary condition for the validity of the Riemann hypothesis for functions that generalize the Riemann zeta-function, Trans. Amer. Math. Soc. 130 (1968), 55-74.

2. J. W. S. Cassels, Footnote to a note of Davenport and Heilbronn, J. London Math. Soc. 36 (1961), 177-184.

3. G. Csordas, T. S. Norfolk, and R. S. Varga, The Riemann hypothesis and the Turán inequalities, Trans. Amer. Math. Soc. 296 (1986), 521-541.

4. H. Davenport and H. Heilbronn, On the zeros of certain Dirichlet series. I, II, J. London Math. Soc. 11 (1936), 181-185, 307-312.

5. N. G. de Bruijn, The roots of trigonometric integrals, Duke Math. J. 17 (1950), 197-226.

6. J. Grommer, Ganze transcendente Funktionen mit lauter reellen Nullstellen, J. Reine Angew. Math. 144 (1914), 114-165.

7. E. Grosswald, Generalization of a formula of Hayman, and its applications to the study of Riemann's zeta-function, Illinois J. Math. 10 (1966), 9-23.

8. J. Hafner, Zeros on the critical line for Dirichlet series attached to certain cusp forms, Math. Ann. 264 (1983), 21-37.

9. G. H. Hardy, Ramanujan, 3rd ed., Chelsea, New York, 1978.

10. Yu V. Matiyasevich, Yet another machine experiment in support of Riemann's conjecture, Cybernetics 18 (1982), 705.

11. G. Pólya, Über trigonometrische Integrale mit nur reellen Nullstellen, J. Reine Angew. Math. 158 (1927), 6-18.

12. F. Shahidi, On certain L-functions, Amer. J. Math. 103 (1981), 297-355. 
13. R. Spira, The integral representation for the Riemann $\Xi$-function, J. Number Theory 3 (1971), 498-501.

14. E. C. Titchmarsh, The theory of the Riemann zeta-function, 2nd ed., Clarendon Press, Oxford, 1986.

15. A. Wintner, A note on the Riemann $\xi$-function, J. London Math. Soc. 10 (1935), 82-83.

Department of Mathematics, Oklahoma State University, Stillwater, Oklahoma 74078

E-mail address: conrey@hardy.math.okstate.edu

E-mail address: ghosh@hardy.math.okstate.edu 